Gynäkologische Endokrinologie 2020 .

18:97-107

https://doi.org/10.1007/s10304-020-00317-y

Online publiziert: 6. April 2020

(c) Der/die Autor(en) 2020

\section{Wissenschaftliche Leitung}

B. Toth, Innsbruck

M. von Wolff, Bern

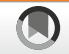

CME

Zertifizierte Fortbildung

\section{Genetische Diagnostik vor assistierter Reproduktion - Empfehlungen der neuen S2k- Leitlinie 2019}

\author{
Sabine Rudnik-Schöneborn ${ }^{1}$ Frank Tüttelmann ${ }^{2}$ Johannes Zschocke \\ ${ }^{1}$ Institut für Humangenetik, Medizinische Universität Innsbruck, Innsbruck, Österreich \\ ${ }^{2}$ Institut für Humangenetik, Universität Münster, Münster, Deutschland
}

\section{Zusammenfassung}

Online teilnehmen unter:

\section{www.springermedizin.de/cme}

Für diese Fortbildungseinheit werden 3 Punkte vergeben.

\section{Kontakt}

Springer Medizin Kundenservice

Tel. 08007780777

(kostenfrei in Deutschland)

E-Mail:

kundenservice@springermedizin.de

\section{Informationen}

zur Teilnahme und Zertifizierung finden Sie im CME-Fragebogen am Ende des Beitrags.
Genetische Ursachen sind für etwa 10-20\% der männlichen und 5-10\% der weiblichen In- oder Subfertilität verantwortlich. Bei Männern ist die andrologische Untersuchung Voraussetzung dafür, genetische Ursachen von Spermatogenesestörungen einordnen zu können. Außer Störungen der Geschlechtschromosomen können auch balancierte Chromosomenumbauten zu einer Fertilitätsminderung führen. Eine Azoospermiefaktoranalyse in Bezug auf Y-chromosomale Mikrodeletionen ist bei Männern mit Azoospermie indiziert, da sie über den Erfolg einer assistierten Reproduktion entscheiden kann. Bei Verdacht auf obstruktive Azoospermie soll eine Analyse des CFTR-Gens zur Diagnostik einer zystischen Fibrose erfolgen. Bei endokrinen Auffälligkeiten sind genetische Ursachen des häufigeren hypergonadotropen Hypogonadismus von denen eines seltenen kongenitalen hypogonadotropen Hypogonadismus zu unterscheiden. Algorithmen zur genetischen Diagnostik sind in der aktuellen Leitlinie im internationalen Vergleich ausgeführt.

\section{Schlüsselwörter}

Weibliche Infertilität · Männliche Infertilität · Techniken der assistierten Reproduktion · Molekularund Zytogenetik · Genetische Beratung 


\section{Lernziele}

Nach Lektüre dieses Beitrags ...

- können Sie die wichtigsten genetischen Ursachen der verminderten Fertilität benennen.

- können Sie auffällige Spermiogrammbefunde möglichen genetischen Störungen zuordnen.

- verstehen Sie die Bedeutung von balancierten Chromosomenumbauten für spätere Schwangerschaften.

- wissen Sie, wie die gestörte Ovarialfunktion im Zusammenhang mit dem Fragiles-X-Syndrom zu beurteilen ist.

- können Sie genetisch-diagnostische Algorithmen bei ungewollter Kinderlosigkeit nach klinischen Vorbefunden ableiten.

\section{Einleitung}

Die Begleitung von Paaren mit unerfülltem Kinderwunsch vor einer Behandlung mit Techniken der assistierten Reproduktion („,assisted reproductive techniques" [ART]) ist eine multidisziplinäre diagnostische und therapeutische Herausforderung. Im Februar 2019 erschien die erste deutschsprachige interdisziplinäre S2kLeitlinie für die „Diagnostik und Therapie vor einer assistierten reproduktionsmedizinischen Behandlung (ART) " [1]. Die Leitlinienerstellung erfolgte im Rahmen des Leitlinienprogramms der Deutschen Gesellschaft für Gynäkologie und Geburtshilfe (DGGG) in Kooperation mit der Schweizerischen Gesellschaft für Gynäkologie und Geburtshilfe (SGGG) und der Österreichischen Gesellschaft für Gynäkologie und Geburtshilfe (OEGGG).

Genetische Ursachen, das heißt Chromosomenveränderungen und monogene Störungen, sind für etwa 10-20\% der männlichen und $5-10 \%$ der weiblichen In- oder Subfertilität verantwortlich. Ziel der Leitlinie ist es, den behandelnden Ärzten evidenzbasierte Empfehlungen zur genetischen Diagnostik vor ART anzubieten.

Im genetischen Abschnitt der Leitlinie haben sich die Verfasser auf genetische Grunderkrankungen konzentriert, aus denen sich

- Konsequenzen für den Erfolg einer ART-Behandlung,

- spezifische ärztliche Vorsorge- bzw. Behandlungsmaßnahmen oder

- Risiken in Bezug auf schwere Erkrankungen, Entwicklungsstörungen oder Behinderungen bei künftigen Kindern

ergeben.

\section{Genetische Ursachen von verminderter Fertilität}

Im Vorfeld einer ART-Behandlung sollte beispielsweise im Rahmen einer genetischen Beratung eine genaue Eigen- und Familienanamnese mit Blick auf mögliche erbliche Belastungen erhoben werden. Insbesondere soll eine mögliche familiäre Häufung von fraglichen genetischen Entwicklungsstörungen, Infertilität oder hormonellen Störungen berücksichtigt werden. Bei Hinweisen auf eine genetische Grunderkrankung in der Familie ist es in der Regel notwendig, bei einer betroffenen Person eine zytogenetische oder molekulargenetische Abklärung vorzunehmen, bevor Risikopersonen getestet werden können.
Genetic diagnosis prior to assisted reproduction: recommendations of the new 2019 S2k guideline

Genetic causes are responsible for around $10-20 \%$ of male and $5-10 \%$ of female infertility or subfertility. In men, an andrological examination is required in order to distinguish different genetic causes of abnormal spermatogenesis. Apart from sex chromosome abnormalities, balanced chromosomal translocation can result in reduced fertility. Azoospermia factor (AZF) analysis to detect $Y$ chromosome deletions is indicated in males with azoospermia, since this can predict the success rate of assisted reproduction techniques (ART). In suspected obstructive azoospermia, analysis of the CFTR gene is recommended to diagnose cystic fibrosis. In terms of genetic causes of endocrine dysfunction, it is important to distinguish between the more frequent hypergonadotropic hypogonadism and the rare congenital hypogonadotropic hypogonadism. Algorithms for genetic diagnostics are discussed in the current guideline in an international context.

Keywords

Infertility, female · Infertility, male · Reproductive techniques, assisted . Molecular and cytogenetics - Genetic counseling

- Expertenkonsens

Bei dem Kinderwunschpaar soll eine genaue Eigen- und Familienanamnese mit Blick auf mögliche genetische Belastungen erhoben werden. Vor einer genetischen Diagnostik soll eine genetische Beratung durch hierfür qualifizierte Ärzte gemäß nationalen Regelungen erfolgen.

Konsensusstärke +++

\section{Genetische Ursachen für Fertilitätsstörungen beim Mann}

Bei bekannter nichtgenetischer Ursache der Spermatogenesestörung (Zustand nach Chemotherapie, Einnahme von anabolen Steroiden) ist gemäß Leitlinie der Weltgesundheitsorganisation (WHO) keine genetische Testung erforderlich [2]. Für die weitere genetische Diagnostik ist es bedeutsam, eine nichtobstruktive Azoospermie bzw. Oligozoospermie von einer obstruktiven Azoospermie zu unterscheiden.

\section{Nichtobstruktive Spermatogenesestörungen}

\section{Mikrodeletionen des $\mathrm{Y}$-Chromosoms}

Mikrodeletionen des Y-Chromosoms stellen die zweihäufigste genetische Ursache einer Spermatogenesestörung dar und haben Bedeutung für die Erfolgschancen einer Hodenbiopsie. Y-Mikrodeletionen werden in Deutschland bzw. Österreich bei weniger als $2 \%$ der infertilen Männer nachgewiesen. Azoospermiefaktorc(AZFc)-Deletionen machen mit etwa $80 \%$ die große Mehrzahl aus und sind mit einem variablen Phänotyp assoziiert. Bei Männern mit Deletionen von AZFa (0,5-4\%), AZFb (1-5\%) sowie AZFbc (1-3\%) kommt es meist zum Sertoli-cell-only-Syndrom. Die Wahrscheinlichkeit, dass in diesen Fällen bei Hodenbiopsien Spermien gewonnen werden können, wird als außerordentlich gering eingeschätzt [3]. In den letzten Jahren wurde jedoch über erfolgreiche Fertilisierungen von AZFb-Deletions-Trägern berichtet [4], sodass zum jetzigen Zeitpunkt noch keine abschließende 
Bei einem ratsuchenden Paar (sie 26, er 28 Jahre alt) bestand seit etwa 3 Jahren ein unerfüllter Kinderwunsch. Gemeinsam hatten die beiden eine gesunde 7-jährige Tochter. Bei den Ratsuchenden waren keine schwerwiegenden Erkrankungen bekannt. Die Menarche war bei der Frau mit etwa 11-12 Jahren eingetreten, die Zyklusanamnese war unregelmäßig und wies auf eine Ovarialinsuffizienz hin. Im Labor zeigten sich Hinweise auf einen hypergonadotropen Hypogonadismus (follikelstimulierendes Hormon 46,4 U/l; $17 \beta$-Östradiol $15 \mathrm{ng} / \mathrm{l}$ ) und eine verminderte ovarielle Reserve (Anti-Müller-Hormon $<0,12 \mu \mathrm{g} / \mathrm{l}$ ). Bei Erhebung der Familienanamnese fiel auf, dass die Schwester der Ratsuchenden mit 29 Jahren in die vorzeitigen Wechseljahre eingetreten war.

Beurteilung zu den Erfolgschancen einer ART-Behandlung bei den unterschiedlichen Deletionen möglich ist.

Bei AZFc-Deletionen reichen die Hodenbefunde von einem Meiosearrest bis zu einer Oligozoospermie aufgrund einer gemischten Atrophie der Spermatogenese [5]. Häufig findet eine residuale Spermatogenese statt, verbunden mit einer durchschnittlichen Erfolgsrate von etwa $50 \%$ für eine Spermiengewinnung mittels testikulärer Spermatozoenextraktion (TESE; [3]). Im AZFc-Bereich finden sich unterschiedliche kleinere Deletionen (unter anderem sogenannte gr/gr-Deletionen), deren diagnostische Relevanz nach jetzigem Wissensstand aber eher von untergeordneter Bedeutung sind, da sie für das weitere Vorgehen bezüglich der ART keine Konsequenzen haben.

\section{Dxpertenkonsens}

Bei nichtobstruktiver Azoospermie soll und bei schwerer Oligozoospermie ( $<5 \mathrm{Mio} . / \mathrm{ml}$ ) sollte nach Ausschluss anderer Ursachen eine Analyse im Hinblick auf AZF-Mikrodeletionen (AZFa, b, c) erfolgen.

Konsensusstärke +++

\section{Chromosomenveränderungen}

Neben Geschlechtschromosomenfehlverteilungen können auch balancierte Chromosomenumbauten zu einer Spermatogenesestörung führen. Dementsprechend ist der Anteil der Männer, die - abgesehen von einer Fertilitätsstörung - gesund sind und eine Chromosomenveränderung tragen, erhöht.

Die Wahrscheinlichkeit von Chromosomenaberrationen bei Männern in Kinderwunschbehandlung steigt bei abnehmender Spermienzahl an (Tab. 1), ist aber bereits bei unauffälligem Spermiogramm gegenüber der Normalbevölkerung erhöht. Dies wird als Subfertilitätsfaktor gedeutet.

In der erwachsenen Allgemeinbevölkerung (Samenspender) wird der Anteil von Chromosomenveränderungen mit 0,3-0,5\% angegeben. Balancierte Chromosomenumbauten sind mit erhöhten Risiken für eine unbalancierte Chromosomenstörung in der Keimbahn verbunden und führen dann zu unterschiedlichen Entwicklungsstörungen bei künftigen Kindern. Die Inzidenz von balancierten Translokationen bzw. Inversionen bei Männern, die eine Behandlung mit intrazytoplasmatischer Spermieninjektion (ICSI) erhielten, war in einer großen französischen Studie [7] um den Faktor 4,5 bzw. 3,3 gegenüber Neugeborenen erhöht. In einer niederländischen Studie wird für Männer mit Fertilitätsstörungen die Rate chromosomaler Strukturanomalien mit 0,9\% angegeben; die-

\begin{tabular}{|l|l|}
\hline $\begin{array}{l}\text { Tab. } 1 \text { Anteil auffälliger Chromosomenanalysen bei Männern mit un- } \\
\text { terschiedlichen Spermiogrammbefunden. (Nach [6]) }\end{array}$ \\
\hline Spermiogrammbefund & $\begin{array}{l}\text { Anteil der Patienten mit } \\
\text { Chromosomenverände- } \\
\text { rungen in \% (Konfidenzin- } \\
\text { tervall) }\end{array}$ \\
\hline Azoospermie $(n=1599)$ & $15,4(13,6-17,2 \%)$ \\
\hline Oligozoospermie $<1 \mathrm{Mio} / \mathrm{ml}(n=539)$ & $3,0(1,5-4,4 \%)$ \\
\hline Oligozoospermie $>1-5 \mathrm{Mio} / \mathrm{ml}(n=475)$ & $2,1(0,8-3,4 \%)$ \\
\hline $\begin{array}{l}\text { Oligozoospermie }>5-10 \mathrm{Mio} . / \mathrm{ml} \\
(n=879)\end{array}$ & $3,5(2,3-4,7 \%)$ \\
\hline $\begin{array}{l}\text { Oligozoospermie }>10-20 \mathrm{Mio} . / \mathrm{ml} \\
(n=808)\end{array}$ & $1,1(0,4-1,8 \%)$ \\
\hline Normozoospermie $>20 \mathrm{Mio} / \mathrm{ml}(n=729)$ & $2,9(1,7-4,1 \%)$ \\
\hline Normalbevölkerung & $0,3-0,5 \%$ \\
\hline
\end{tabular}

se würde zu einem erhöhten Risiko von Chromosomenstörungen bei Kindern führen [6].

In einer aktuellen niederländisch-belgischen Multicenterstudie wurden bei $14,4 \%$ der untersuchten Männer mit Azoospermie ( $n=1663$ ) chromosomale Aberrationen nachgewiesen [8]. Es zeigte sich bezüglich der Therapierelevanz kein signifikanter Unterschied zwischen hypergonadotropen und normogonadotropen Männern.

\section{- Expertenkonsens}

Bei nichtobstruktiver Azoospermie oder schwerer Oligozoospermie ( $<5$ Mio./ml) soll nach Ausschluss anderer Ursachen eine Chromosomenanalyse erfolgen.

\section{Konsensusstärke +++}

\section{Monogene Spermatogenesestörungen}

Da die Ursache nichtobstruktiver Spermatogenesestörungen bei über $80 \%$ der betroffenen Männer bisher nicht bekannt ist, wird intensiv nach neuen Kandidatengenen gesucht. In den letzten Jahren sind seltene monogene Spermatogenesestörungen, die beispielsweise eine bestimmte Spermienmorphologie zur Folge haben, identifiziert worden und können grundsätzlich identifiziert werden, unter anderem über Multigenpanels [9, 10].

Beim Angebot von genetischen Analysen zu monogenen Spermatogenesestörungen ist zu berücksichtigen, dass nachgewiesene Störungen bislang keinen Einfluss auf das Verfahren oder den Ausgang der ART haben. Die Beurteilung kann sich in der Zukunft ändern, wenn sich in Abhängigkeit von der genetischen Ursache gezielte Behandlungen ergeben. Weitere wissenschaftliche Studien sind erforderlich, um die Bedeutung einzelner genetischer Mutationen für die Spermatogenese besser einordnen zu können.

\footnotetext{
$>$ Expertenkonsens
}

Bei Verdacht auf eine seltene monogene Spermatogenesestörung kann eine genetische Analyse angeboten werden.

Konsensusstärke +++ 


\section{Obstruktive Azoospermie und zystische Fibrose}

Grundlage für diese Diagnose ist eine intakte Spermatogenese im Hodengewebe, die gute Erfolgschancen für eine ART im Rahmen einer TESE/ICSI bedeutet. Etwa 2\% der Männer mit einer Azoospermie haben eine Fehlanlage der Derivate des WolffGangs, meist in Form einer kongenitalen bilateralen Aplasie des Vas deferens (CBAVD), seltener durch eine bilaterale Nebenhodenobstruktion.

Bei etwa $80 \%$ der Patienten mit CBAVD wird mindestens eine Cystic-fibrosis-transmembrane-conductance-regulator(CFTR)Mutation gefunden und als Ursache der CBAVD eine milde Form der zystischen Fibrose (CF) bzw. eine sogenannte CF-assoziierte Erkrankung angenommen [11].

Die CF ist eine der häufigsten autosomal-rezessiv vererbten Stoffwechselerkrankungen und tritt bei etwa einer von 3300 Personen auf. Das klinische Spektrum der Erkrankung, die durch eine erhöhte Viskosität des Sekrets exokriner Drüsen gekennzeichnet ist, reicht von schwer betroffenen Neugeborenen mit Mekoniumileus über Kinder mit Lungen- und Gedeihstörungen bis hin zu praktisch symptomlosen Männern, die nur durch eine Azoospermie auffallen.

Wird bei einem Mann mindestens eine klassische CFTR-Mutation nachgewiesen, besteht die Indikation zur genetischen Testung seiner Partnerin. In der mitteleuropäischen Bevökerung trägt etwa jede 30. Person eine heterozygote CFTR-Mutation. Ist ein Partner von einer CF betroffen und der andere Partner heterozygoter Träger einer CFTR-Mutation, besteht ein Risiko von $50 \%$ für ein Kind mit CF, ohne dass der Schweregrad der Erkrankung vorhergesagt werden könnte. Tragen beide Partner eine CFTR-Mutation, ist es wichtig, die Konsequenzen einer ART im Rahmen einer genetischen Beratung zu besprechen und auf die Möglichkeit einer pränatalen und präimplantativen genetischen Diagnostik hinzuweisen.

CFTR-Mutationen sind nur wirksam, wenn sie beide chromosomalen Allele betreffen. Es gibt bisher keine sichere Evidenz dafür, dass auch heterozygote Anlageträger eine erhöhte Rate an urogenitalen Fehlanlagen oder Fertilitätsstörungen aufweisen. Die den meisten Studien und Empfehlungen zugrunde liegenden Untersuchungsverfahren beschränken sich auf das Screening ausgewählter Mutationen ("CFTR-Testkits") mit einer maximalen Erfassungsrate von 80 bis $95 \%$ für CF-spezifische Mutationen bzw. nur etwa $60 \%$ für Mutationen, die CF-assoziierte Erkrankungen wie die CBAVD verursachen.

Erst mit der Implementierung der massiven parallelen Sequenzierung wurde eine kostengünstige Analyse aller codierenden Abschnitte des CFTR-Gens möglich. Für Patienten mit CBAVD, bei denen nur eine Mutation festgestellt wurde, ist im Nachhinein nicht zu beurteilen, ob eine zweite, nicht erkannte, Mutation vorgelegen hat, die das klinische Bild begründete. Wenngleich bisher nur wenige Studien mit größeren Patientenserien und neueren Sequenzierverfahren veröffentlicht sind, lassen erste Daten doch den Schluss zu, dass bei obstruktiver Azoospermie eine komplette Sequenzierung des CFTR-Gens sinnvoll ist, um pathogene Mutationen, die zu einer klassischen oder atypischen CF führen können (einschließlich der T-Allele), vollständig zu erfassen. In Anbetracht des CF-Risikos bei künftigen Kindern schafft nur eine vollständige
CFTR-Analyse bei Männern mit obstruktiver Azoospermie ausreichende Beratungssicherheit.

$>$ Expertenkonsens

Bei Verdacht auf eine obstruktive Azoospermie soll nach Ausschluss anderer Ursachen eine Analyse des CFTR-Gens erfolgen. Diese soll alle relevanten pathogenen Mutationen inklusive des TG-T-Repeats in Intron 8 erfassen; falls damit nur eine heterozygote Mutation gefunden wird, soll eine vollständige Sequenzierung erfolgen.

Konsensusstärke +++

Bei weniger als $5 \%$ der Männer mit obstruktiver Azoospermie bestehen zusätzlich einseitige Nierenfehlanlagen (Nierendysplasie oder Nierenagenesie) im Sinne einer kombinierten Entwicklungsstörung der Derivate des Wolff-Gangs. Aus diesem Grund ist bei Männern mit einer obstruktiven Azoospermie eine Ultraschalluntersuchung des Harntrakts indiziert [2].

Inzwischen ist hinreichend belegt, dass eine obstruktive Azoospermie in Verbindung mit Fehlanlagen der Niere nicht durch CFTR-Mutationen bedingt wird und auf andere genetische oder nichtgenetische Faktoren zurückzuführen ist [11]. Mit dem Gen ADGRG2 wurde 2016 eine X-chromosomale Ursache einer CBAVD identifiziert [12]. Ersten Studien zufolge weisen etwa 20\% (4 von 26) der Männer mit CBAVD ohne Nierenfehlbildungen und ohne Nachweis einer CFTR-Mutation Loss-of-function-Mutationen in $A D G R G 2$ auf, während bei Männern mit Nierenfehlbildungen $(n=28)$ keine Mutationen festgestellt wurden [12].

\section{- Expertenkonsens}

Sofern bei einer obstruktiven Azoospermie die CFTR-Analyse einen unauffälligen Befund erbracht hat, sollte eine Analyse des Gens ADGRG2 erfolgen.

Konsensusstärke +++

\section{Endokrine Störungen}

Bei endokrinen Auffälligkeiten kommt der Differenzierung zwischen hypo- und hypergonadotropem Hypogonadismus bezüglich der genetischen Untersuchung eine wichtige Rolle zu.

\section{Klinefelter-Syndrom und hypergonadotroper}

Hypogonadismus

Eine primäre testikuläre Funktionsstörung findet sich vor allem beim Klinefelter-Syndrom, das mit fast $14 \%$ bei Männern mit Azoospermie die häufigste genetische Ursache der männlichen Infertilität darstellt. Bei $80 \%$ liegt ein Karyotyp 47,XXY vor, bei etwa 20\% bestehen höhergradige Aneuploidien, Mosaike mit 46,XX/47,XXY oder strukturell veränderte X-Chromosomen [13]. Hierbei handelt es sich in der Regel um Aberrationen, die einer mikroskopischen Chromosomenanalyse zugänglich sind.

Bei über $90 \%$ der Männer mit Klinefelter-Syndrom besteht eine Azoospermie, bei weniger als $10 \%$ der Betroffenen werden einzelne Spermien mit verminderter Motilität und Morphologie gefunden [14]. Mehrheitlich kommt es im Verlauf zu einem hypergonadotropen Hypogonadismus. 
Die molekulargenetische Analyse der CGG-Trinukleotid-Repeats in FMR1 ergab bei der Ratsuchenden ein Normalallel mit etwa $30 \pm 2$ Repeats und ein Allel im Prämutationsbereich mit etwa 80 Repeats. Nachfolgend wurde auch bei der Schwester ein vergleichbarer Befund erhoben, die Prämutation stammte vom Vater. Die Ratsuchenden wurden darüber aufgeklärt, dass sich bei 80-90 CGG-Repeats das Risiko einer Vollmutation im Falle der Weitergabe des mutierten X-Chromosoms auf etwa 70-80\% beläuft. Damit betrug das Risiko eines Fragiles-X-Syndroms bei künftigen Söhnen etwa 30-40\%, bei künftigen Töchtern etwa 10-15\%. Bei der Patientin wurde mit hormoneller Stimulation eine Schwangerschaft erzielt. Die werdenden Eltern entschieden sich für eine Chorionzottenbiopsie. In der kindlichen DNA-Probe zeigte sich ein FMR1-Allel im Prämutationsbereich von etwa 84 Repeats. Damit hatte der ungeborene Sohn der Ratsuchenden kein Risiko für ein Fragiles-X-Syndrom, das heißt, bei der Vererbung von Mutter zu Sohn hatte keine Zunahme der CGG-Repeats stattgefunden. Die Schwangerschaft wurde fortgesetzt.

Die Behandlung der Infertilität beim Klinefelter-Syndrom hat sich seit den 2000er-Jahren drastisch geändert, nachdem erfolgreiche TESE- und ICSI-Behandlungen trotz Azoospermie durchgeführt wurden. Nach einer Metaanalyse [15] beträgt die kumulative Spermiengewinnungsrate pro TESE-Zyklus bei Männern mit 47,XXYKaryotyp etwa $44 \%$. Die kumulative Lebendgeburtenrate pro ICSI-Zyklus wurde in der genannten Metaanalyse mit $43 \%$ angegeben. Es zeigte sich kein Zusammenhang zwischen Alter, Hodenvolumen, den Werten von Testosteron oder luteinisierendem Hormon der Patienten und der Erfolgsrate der ART-Behandlung [15].

\section{- Expertenkonsens}

Bei Männern mit hypergonadotropem Hypogonadismus soll nach Ausschluss anderer Ursachen eine Chromosomenanalyse durchgeführt werden.

\section{Konsensusstärke +++}

\section{Hypogonadotroper Hypogonadismus}

Ein kongenitaler hypogonadotroper Hypogonadismus $(\mathrm{CHH})$ ist mit etwa 1 zu 4000-10.000 Männern ungefähr 3- bis 5-fach häufiger als bei Frauen [16]. Es wird formal der normosmische hypogonadotrope Hypogonadismus vom Kallmann-Syndrom unterschieden, bei dem der Geruchsinn herabgesetzt ist; die Übergänge sind jedoch fließend. Als Ursache kommen zahlreiche Gene in Betracht, die in der Summe etwa 40-50\% der Fälle erklären [17] und von denen das X-chromosomale Gen KAL1 mit etwa $10 \%$ die wichtigste Rolle spielt.

Die Beurteilung von genetischen Befunden wird durch additive Effekte mehrerer Mutationen erschwert. Bei etwa 20\% der Patienten werden Mutationen in mehreren Genen (oligogene Vererbung) nachgewiesen, das heißt, die Frage des verantwortlichen Erbgangs ist dann keineswegs klar zu beantworten. Zudem gibt es keine genetische Diagnose, die für die ART eine bestimmte Prognose oder ein unterschiedliches Therapieregime ausweist. Bei 50-60\% der Patienten mit normosmischem hypogonadotropem Hypogonadismus werden erfolgreiche Fertilisationen mittels ICSI erzielt, darüber hinaus kommt es bei 10-20\% der Patienten zu einer spontanen Normalisierung der hormonellen Situation [17].
- Expertenkonsens

Bei Männern mit einem CHH kann nach Ausschluss exogener Ursachen eine genetische Analyse der $\mathrm{CHH}-G e n e$ durchgeführt werden Konsensusstärke +++

\section{Genetische Ursachen für Fertilitätsstörungen bei der Frau}

\section{Ovulatorische Dysfunktion}

Bei etwa $40 \%$ der Frauen mit Fertilitätsstörung besteht eine Oligobzw. Amenorrhö (ovulatorische Dysfunktion). Der entscheidende Faktor - auch für den Erfolg einer ART mit eigenen Oozyten ist das mütterliche Alter, während primär genetische Ursachen vergleichsweise selten sind. Ab einem Alter von 40 bis 45 Jahren ist die große Mehrzahl der Oozyten aneuploid, sodass sich nur noch ein kleiner Prozentsatz erfolgreich zur Blastozyste entwickelt und implantiert $[18,19]$.

\section{Endokrine Funktionsstörungen}

Bei der Untersuchung der hormonellen Achsen wird insbesondere geprüft, ob ein hypo- oder hypergonadotroper Hypogonadismus oder eine Hyperandrogenämie vorliegt.

\section{Hypergonadotroper Hypogonadismus/Turner-Syndrom}

Bei 10-13\% der betroffenen Frauen liegt ein Turner-Syndrom aufgrund einer Gonosomenaberration mit einer 45,X-, oder 47, XXXZelllinie oder einem strukturell veränderten $X$-Chromosom vor. Bei ovarieller Funktionsstörung ist eine Chromosomenanalyse indiziert, sofern keine anderen Ursachen in Betracht kommen [20]. Weiterbetreuung und Therapie richten sich nach dem genetischen Befund. Bei 45,X/46,XX-Mosaiken besteht eine inverse Korrelation zwischen dem prozentualen Anteil einer 45,X-Zelllinie und der Wahrscheinlichkeit für eine normale Pubertät, spontane Zyklen und Fertilität [21]. Ein geringgradiges Mosaik im Blut mit einer $45, X$-Zelllinie unter $30 \%$ scheint die Ovarialfunktion nicht einzuschränken [22].

Bei erwachsenen Frauen mit Turner-Syndrom und Kinderwunsch ist im Falle einer primären Amenorrhö auf der Grundlage eines durchgehenden 45,X-Karyotyps nicht mit der Gewinnung fertilisierbarer Oozyten zu rechnen [23]. Da zu Beginn der Pubertät eine ovarielle Reserve vorhanden sein kann, schließen aktuelle Empfehlungen eine Eizellentnahme und Kryokonservierung bei Mädchen mit Turner-Syndrom ab einem Alter von 12 bis 13 Jahren

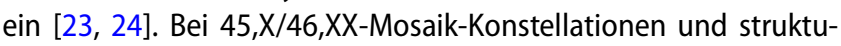
rell veränderten $X$-Chromosomen richtet sich die ART-Behandlung nach dem klinischen und endokrinologischen Befund. Frauen mit Turner-Syndrom, die eine Eizellspende in Anspruch nehmen, haben eine verminderte Lebendgeburtenrate, eine hohe Abortrate und ein erhöhtes Risiko von Schwangerschaftskomplikationen, die bei der Beratung vor ART angesprochen werden müssen [25].

Die Fruchtbarkeit wird bei Frauen mit Trisomie X $(47, X X X)$ als normal eingestuft, aber es besteht ein erhöhtes Risiko der primären Ovarialinsuffizienz [26]. Ein auffälliger Chromosomenbefund und seine Konsequenzen für die Betroffene und ihre Familienplanung sollten im Rahmen einer genetischen Beratung diskutiert werden. 
Karyotyp: 46,XX,t(2;6)(q?31;q?23)

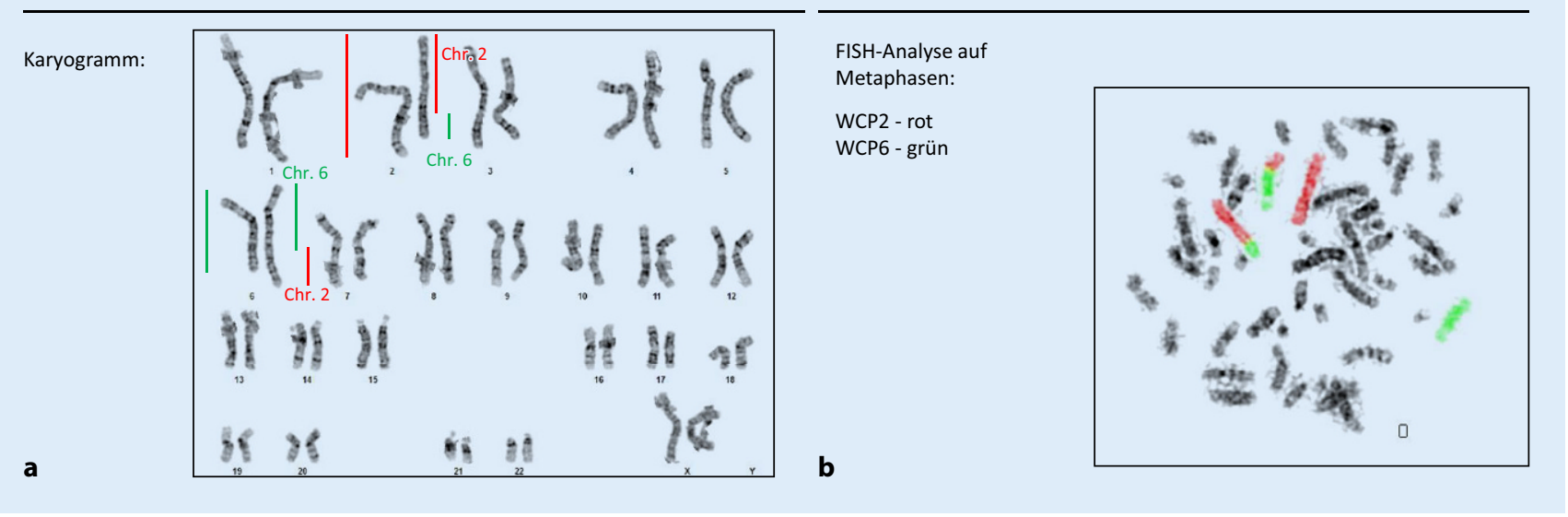

Abb. 1 ॥ Karyogramm und FISH-Analyse bei einer balancierten Translokation. (Freundlicherweise zur Verfügung gestellt von Frau Dr. Birgit Nachbauer, Institut für Humangenetik, Medizinische Universität Innsbruck, Österreich). a Es zeigen sich strukturelle Auffälligkeiten an jeweils einem Chromosom 2 und 6 bei der Frau. $\mathbf{b}$ Die FISH-Analyse weist eine reziproke Translokation zwischen den langen Armen der Chromosomen 2 und 6 mit der Karyotypformel 46,XX,t(2;6)(q?31;q?23) aus. Damit besteht bei der Eizellbildung der Frau ein hohes Risiko einer unbalancierten Translokation. FISH Fluoreszenz-in-situ-Hybridisierung

\section{Expertenkonsens}

Bei Frauen mit hypergonadotropem Hypogonadismus soll nach Ausschluss anderer Ursachen eine Chromosomenanalyse durchgeführt werden.

\section{Konsensusstärke +++}

\section{Fragiles-X-Syndrom, FMR1-Prämutationen}

Prämutationen im Gen FMR1 führen gehäuft zu einer primären oder sekundären Ovarialinsuffizienz und haben bei der Weitergabe an Kinder eine hohe Wahrscheinlichkeit, zu einer Vollmutation zu expandieren. Während nur etwa $1 \%$ der Frauen in der Normalbevölkerung vor dem 40. Lebensjahr keine Zyklen mehr hat, betrifft diese Störung etwa 20-25\% der Frauen mit FMR1-Prämutation. Das Fragiles-X-Syndrom ist die wichtigste Form der geschlechtsgebunden vererbten geistigen Behinderung und tritt mit einer Häufigkeit von etwa 1 zu 4000-5000 in der Bevölkerung auf [27].

Die Ursache des Fragiles-X-Syndroms ist eine Erbgutveränderung (Trinukleotid-Repeat-Vermehrung) in FMR1 auf dem X-Chromosom. Normalerweise liegen nicht mehr als 44 CGG-Repeats hintereinander vor. Wenn eine Repeat-Zahl von 200 überschritten wird, kommt es zu einer Vollmutation, bei der FMR1 nicht mehr exprimiert wird. In der Folge werden Entwicklung und Funktion des Gehirns gestört. Eine Anzahl zwischen 55 und 200 CGG-Repeats wird als Prämutation bezeichnet. In Abhängigkeit von der Länge der mütterlichen Prämutation expandiert die Repeat-Zahl bei der Weitergabe an ein Kind, bis schließlich eine Vollmutation auftritt. Das veränderte X-Chromosom wird von Prämutationsträgerinnen an die Hälfte der Kinder vererbt. Eine Repeat-Expansion von einer Prämutation zu einer Vollmutation bei der Weitergabe an ein Kind wird nur bei Frauen beobachtet, während männliche Prämutationsträger in der Regel nur stabile Prämutationen an ihre Töchter vererben.

Etwa $85 \%$ der männlichen Träger und 25-30\% der weiblichen Träger einer FMR1-Vollmutation weisen eine geistige Behinderung auf (Fragiles-X-Syndrom). FMR1-Vollmutationen sind nicht mit einer Ovarialinsuffizienz assoziiert. Kaukasische Frauen mit primärer Ovarialinsuffizienz weisen zu etwa $2 \%$ bei Fällen ohne familiäre Häufung und zu 10-15\% bei familiärer Häufung eine Prämutation in FMR1 auf [28].

\section{- Expertenkonsens}

Bei primärer oder prämaturer Ovarialinsuffizienz soll nach Ausschluss anderer Ursachen eine genetische Analyse der CGG-Wiederholungen in FMR1 durchgeführt werden.

Konsensusstärke +++

\section{Hypogonadotroper Hypogonadismus}

Ein CHH ist mit einer Häufigkeit von etwa 1 zu 30.000-40.000 Frauen sehr selten. Inzwischen sind 35-40\% der molekularen Ursachen des $\mathrm{CHH}$ bekannt und auf Mutationen in mindestens 20 Genen zurückzuführen [29]. Die exogene Zufuhr von Geschlechtshormonen, Gonadotropinen bzw. Gonadotropin-Releasing-Hormon-Analoga stellt bei allen bekannten Störungen die einzige Therapieoption dar.

\section{$>$ Expertenkonsens}

Bei Frauen mit CHH kann nach Ausschluss exogener Ursachen eine genetische Analyse der $\mathrm{CHH}-\mathrm{Gene}$ durchgeführt werden.

Konsensusstärke +++

\section{Hyperandrogenämie/adrenogenitales Syndrom}

Die wichtigste genetische Grunderkrankung, die zu einer Hyperandrogenämie führt, ist das adrenogenitale Syndrom (AGS). Die häufigste Form ist der 21-Hydroxylase-Mangel bedingt durch autosomal-rezessive Mutationen im Gen CYP21A2. Frauen mit milden CYP21A2-Mutationen oder einer Kombination aus einer klassischen und einer milden Mutation entwickeln kein Vollbild des AGS, sondern unterschiedliche Merkmale des Late-onset-AGS mit Zyklus- 
Tab. 2 Vergleich der Empfehlungen verschiedener Fachgesellschaften für die genetische Diagnostik bei Paaren vor ART (nach Jahr der Veröffentlichung sortiert)

\begin{tabular}{|c|c|c|c|c|c|}
\hline & \begin{tabular}{|l|}
$\begin{array}{l}\text { Foresta et al. } 2002 \\
\text { (Italien; [32]) }\end{array}$ \\
\end{tabular} & $\begin{array}{l}\text { ACOG 2005/2015 (USA; } \\
\text { [33]) }\end{array}$ & $\begin{array}{l}\text { RCOG } 2013 \text { (England; } \\
{[34] \text { ) }}\end{array}$ & $\begin{array}{l}\text { SOGC } 2014 \text { (Kanada; } \\
{[35] \text { ) }}\end{array}$ & ASRM 2015 (USA; [36]) \\
\hline \multicolumn{6}{|c|}{ Untersuchungen beim Mann } \\
\hline $\begin{array}{l}\text { Karyo- } \\
\text { typisierung }\end{array}$ & Empfohlen & Nicht empfohlen & Empfohlen & $\begin{array}{l}\text { Empfohlen bei nichtob- } \\
\text { struktiver Azoosper- } \\
\text { mie, Oligozoospermie } \\
<5 \text { Mio./ml }\end{array}$ & Keine Angaben \\
\hline $\begin{array}{l}\text { Y-Mikrodele- } \\
\text { tions-Analyse }\end{array}$ & \begin{tabular}{|l|} 
Empfohlen bei Oligo- \\
zoospermie $<10$ Mio./ml
\end{tabular} & $\begin{array}{l}\text { Empfohlen bei Oligo- } \\
\text { zoospermie }<5 \text { Mio./ml }\end{array}$ & Nicht empfohlen & $\begin{array}{l}\text { Empfohlen bei nichtob- } \\
\text { struktiver Azoosper- } \\
\text { mie, Oligozoospermie } \\
<5 \text { Mio./ml }\end{array}$ & $\begin{array}{l}\text { Empfohlen bei nichtob- } \\
\text { struktiver Azoosper- } \\
\text { mie, Oligozoospermie } \\
<5 \text { Mio./ml }\end{array}$ \\
\hline CFTR-Analyse & Empfohlen bei CBAVD & Empfohlen bei CBAVD & Empfohlen bei CBAVD & $\begin{array}{l}\text { Empfohlen bei obstruk- } \\
\text { tiver Azoospermie }\end{array}$ & $\begin{array}{l}\text { Empfohlen bei CBAVD/ } \\
\text { CUAVD/bilateraler } \\
\text { Nebenhodenobstruktion }\end{array}$ \\
\hline KAL1-Analyse & $\begin{array}{l}\text { Empfohlen bei Azoo- } \\
\text { spermie mit } \mathrm{nHH} / \mathrm{KS}\end{array}$ & k.A. & k. A. & k. A. & k. A. \\
\hline \multicolumn{6}{|c|}{ Untersuchungen bei der Frau } \\
\hline $\begin{array}{l}\text { Karyo- } \\
\text { typisierung }\end{array}$ & Empfohlen & k. A. & Empfohlen & k. A. & k. A. \\
\hline $\begin{array}{l}\text { FMR1- } \\
\text { Prämutation }\end{array}$ & $\begin{array}{l}\text { Empfohlen bei Verdacht } \\
\text { auf POI und "poor re- } \\
\text { sponder" }\end{array}$ & k.A. & k. A. & k. A. & k. A. \\
\hline KAL1-Analyse & Empfohlen bei $\mathrm{nHH} / \mathrm{KS}$ & k.A. & k. A. & k. A. & k. A. \\
\hline CFTR-Analyse & Empfohlen & k. A. & k. A. & k. A. & k. A. \\
\hline \multicolumn{6}{|c|}{$\begin{array}{l}\text { ACOG American College of Obstetricians and Gynecologists, } A R T \text {,assisted reproductive techniques" (Techniken der assistierten Reproduktion), ASRM Amer- } \\
\text { ican Society for Reproductive Medicine, CBAVD kongenitale bilaterale Aplasie des Vas deferens, CUAVD kongenitale unilaterale Aplasie des Vas deferens, } \\
\text { k. A. keine Angaben, KS Kallmann-Syndrom, } n H H \text { normosmischer hypogonadotroper Hypogonadismus, POI primäre Ovarialinsuffizienz, RCOG Royal } \\
\text { College of Obstetricians and Gynecologists, SOGC Society of Obstetricians and Gynaecologists of Canada } \\
\text { aNicht bei CBAVD/CUAVD mit Nierendysplasie }\end{array}$} \\
\hline
\end{tabular}

störungen und Zeichen einer Hyperandrogenämie. Wird bei einer Frau mit Hyperandrogenämie eine klassische CYP21A2-Mutation nachgewiesen, ist die Indikation zur genetischen Untersuchung des Partners gegeben. Die Heterozygotenrate für das AGS in der Bevölkerung liegt bei 1 zu 65. Sind beide Eltern Träger einer klassischen CYP21A2-Mutation, haben künftige Kinder ein erhöhtes AGS-Risiko. Die pränatale Therapie zur Vermeidung einer Virilisierung bei weiblichen Feten mit einem AGS-Risiko auf Grundlage eines 21-Hydroxylase-Mangels wurde zum Zeitpunkt der Leitlinienerstellung als experimentell eingestuft und sollte deshalb im Einzelfall sorgfältig abgewogen werden [30].

\section{- Expertenkonsens}

Bei Verdacht auf ein AGS soll eine genetische Diagnostik durchgeführt werden.

\section{Konsensusstärke ++}

\section{Balancierte Chromosomenveränderungen bei der Frau}

Strukturelle Chromosomenaberrationen stellen auch bei Frauen eine relevante Ursache für einen unerfüllten Kinderwunsch dar, ohne dass sich bei gynäkologischen Untersuchungen Auffälligkeiten ergeben (Abb. 1). In einer französischen Studie zeigten die chromosomal untersuchten Partnerinnen von infertilen Männern einen vergleichbar erhöhten Anteil an balancierten Translokationen (Faktor 4,5) bzw. Inversionen (Faktor 16) wie die untersuchten
Männer ( $n=3208$ Patienten; [7]). Der Prozentsatz an Frauen mit Chromosomenveränderungen korrelierte hierbei invers mit dem pathologischen Spermiogrammbefund des Partners. In einer italienischen Studie wurden balancierte strukturelle Chromosomenveränderungen bei 0,9\% der Männer und 0,5\% der Frauen gefunden; sie waren damit um den Faktor 2-3 gegenüber Neugeborenen erhöht [31]. In einer Übersicht aus den Niederlanden wurden für die weiblichen Partner von ICSI-Paaren Raten chromosomaler Auffälligkeiten zwischen 3,4 und 4,9\% angegeben [6].

$>$ Expertenkonsens

Nach Ausschluss anderer Ursachen für die Infertilität sollte eine Chromosomenanalyse beider Partner durchgeführt werden.

Konsensusstärke +

\section{Therapieoptionen im Falle eines balancierten Chromosomenumbaus bei einem Partner}

Eine ursächliche Therapie von Chromosomenaberrationen ist nicht möglich. Wird bei einem der Partner eine balancierte Chromosomenveränderung nachgewiesen, erhöht sich in Abhängigkeit von den beteiligten Chromosomen das Risiko für Aborte oder für die Geburt eines Kindes mit einer unbalancierten Chromosomenstörung. Das Risiko einer kindlichen Entwicklungsstörung aufgrund einer unbalancierten Chromosomenaberration wird insgesamt als sehr gering eingestuft, je nach Chromosomenbefund kann es aber 


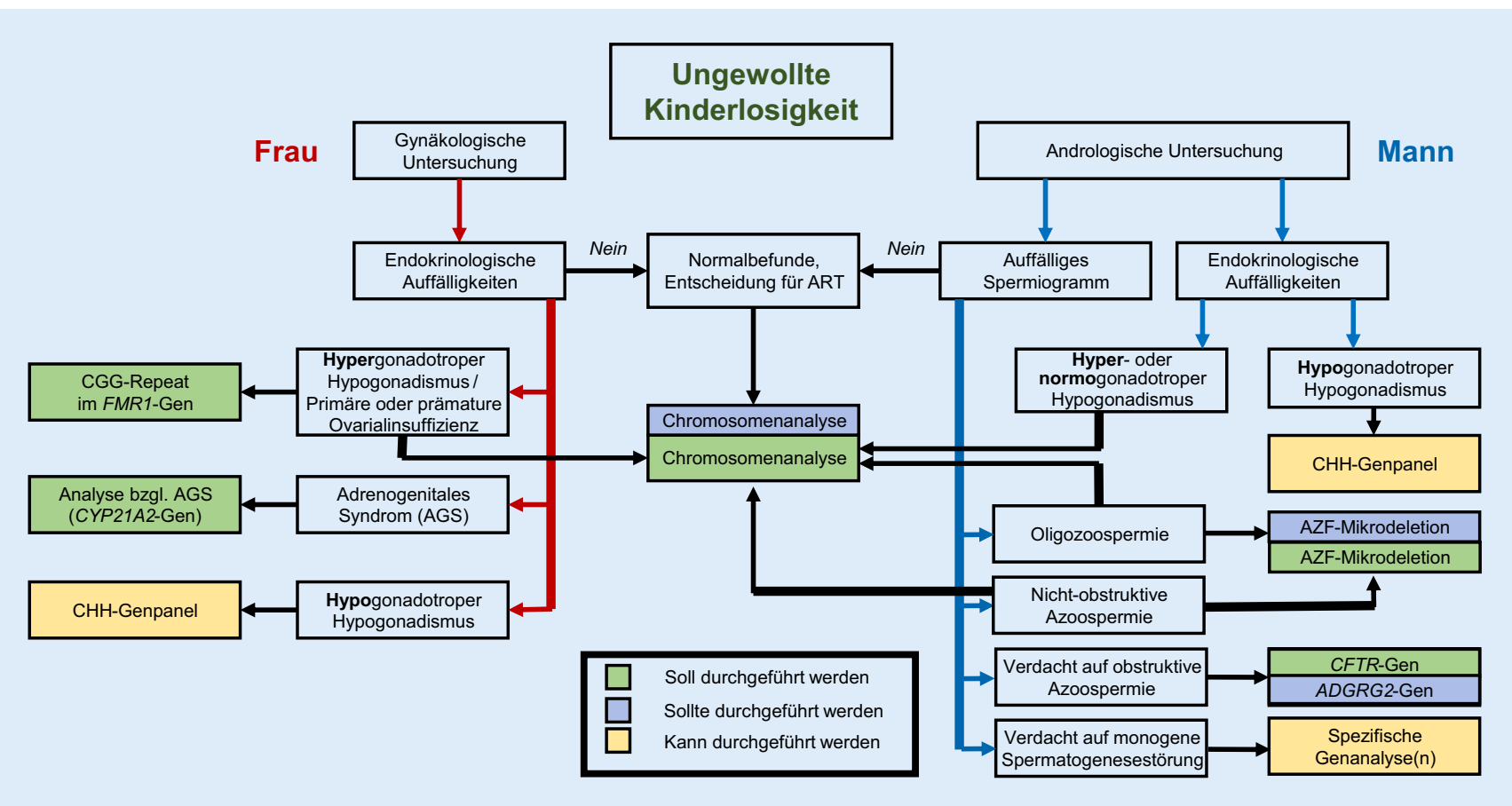

Abb. 2 ム Zusammenfassung der Empfehlungen zur genetischen Diagnostik bei Paaren vor ART [1]. ART „Assisted reproductive techniques“ (Techniken der assistierten Reproduktion), AZF Azoospermiefaktor, AGS adrenogenitales Syndrom, CHH kongenitaler hypogonadotroper Hypogonadismus

zu schwerwiegenden Behinderungen kommen, einhergehend mit erheblichen Belastungen für das betroffene Paar [8]. Hieraus ergeben sich Konsequenzen für das Angebot einer pränatalen Diagnostik in weiteren Schwangerschaften.

\section{$>$ Expertenkonsens}

Bei Nachweis einer strukturellen Chromosomenveränderung bei einem Partner soll das Paar über die Möglichkeiten einer Polkörper- und Präimplantationsdiagnostik sowie der vorgeburtlichen Diagnostik (invasive und nichtinvasive Pränataldiagnostik) informiert werden.

\section{Konsensusstärke +++}

\section{Empfehlungen anderer Fachgesellschaften}

Zur Klärung der Chancen einer ART und möglicher genetischer Risiken für Nachkommen werden nach Auffassung der meisten Fachgesellschaften und Arbeitsgruppen eine Chromosomenanalyse beider Partner und in Abhängigkeit vom andrologischen und gynäkologischen Befund weitere genetische Analysen empfohlen (Tab. 2). Diese Empfehlungen waren Grundlage für die Erstellung der aktuellen Leitlinie mit den hierin zusammengefassten diagnostischen Algorithmen (Abb. 2).

\section{Fazit für die Praxis}

- Genetische Ursachen sind für 10-20\% der männlichen und 5-10\% der weiblichen Infertilität verantwortlich.

- Der wichtigste genetische Risikofaktor für den Erfolg einer assistierten Reproduktion ist das mütterliche Alter.
- Bei 10-15\% der Männer mit nichtobstruktiver Spermiogenesestörung liegen Störungen der Geschlechtschromosomen und balancierte Chromosomenumbauten vor.

- Mikrodeletionen des Y-Chromosoms werden bei etwa $2 \%$ der Männer mit Azoospermie nachgewiesen.

- CFTR-Mutationen führen zur obstruktiven Azoospermie und zystischen Fibrose.

- Häufigste genetische Ursache des hypergonadotropen Hypogonadismus beim Mann ist das Klinefelter-Syndrom, bei der Frau ist es das Turner-Syndrom.

- Frauen mit primärer Ovarialinsuffizienz weisen zu etwa $2 \%$ bei Fällen ohne familiäre Häufung und zu 10-15\% bei familiärer Häufung eine Prämutation im FMR1-Gen auf.

- Beim seltenen kongenitalen hypogonadotropen Hypogonadismus (CHH) sind Mutationen in zahlreichen CHH-Genen ursächlich.

- Bei ungeklärter Infertilität eines Paars ist eine Chromosomenanalyse sinnvoll, um eine balancierte Chromosomenstörung eines Partners aufzudecken, aus der sich für die pränatale Diagnostik Konsequenzen ergeben.

\section{Korrespondenzadresse}

Prof. Dr. med. Sabine Rudnik-Schöneborn

Institut für Humangenetik, Medizinische Universität Innsbruck Peter-Mayr-Str. 1, 6020 Innsbruck, Österreich sabine.rudnik@i-med.ac.at

Funding. Open access funding provided by University of Innsbruck and Medical University of Innsbruck. 


\section{Einhaltung ethischer Richtlinien}

Interessenkonflikt. Gemäß den Richtlinien des Springer Medizin Verlags werden Autoren und Wissenschaftliche Leitung im Rahmen der Manuskripterstellung und Manuskriptfreigabe aufgefordert, eine vollständige Erklärung zu ihren finanziellen und nichtfinanziellen Interessen abzugeben.

Autoren. S. Rudnik-Schöneborn gibt an, dass kein finanzieller Interessenkonflikt besteht. Nichtfinanzielle Interessen: Professorin und Fachärztin für Humangenetik, Universität Innsbruck, Österreich | Mitgliedschaften: Österreichische Gesellschaft für Humangenetik (ÖGH), Deutsche Gesellschaft für Humangenetik (GfH). F. Tüttelmann gibt an, dass kein finanzieller Interessenkonflikt besteht. Nichtfinanzielle Interessen: Professor für Reproduktionsgenetik, Universität Münster|Mitgliedschaften: European Society of Human Genetics (ESHG), Deutsche Gesellschaft für Humangenetik $(\mathrm{GfH})$, European Society of Human Reproduction (ESHRE), European Academy of Andrology (EAA), Deutsche Gesellschaft für Reproduktionsmedizin (DGRM), Deutsche Gesellschaft für Andrologie (DGA) | Vorstandsmitglied: International Male Infertility Genomics Consortium (IMIGC), European Academy of Andrology (EAA), Deutsche Gesellschaft für Reproduktionsmedizin (DGRM). J. Zschocke: Finanzielle Interessen: akademische Förderung (nicht im Bereich des Manuskripts bzw. der Reproduktionsmedizin). - Kostenerstattung für Vorträge zur genetischen Diagnostik in der Reproduktionsmedizin. - bezahlter Berater/interner Schulungsreferent/Gehaltsempfänger o. Ä.:nicht im Bereich des Manuskripts bzw. der Reproduktionsmedizin. Nichtfinanzielle Interessen: Institutsleiter, Medizinische Universität Innsbruck| Mitglied zahlreicher Fachgesellschaften und relevanter Fachgremien.

Wissenschaftliche Leitung. Die vollständige Erklärung zum Interessenkonflikt der Wissenschaftlichen Leitung finden Sie am Kurs der zertifizierten Fortbildung auf www. springermedizin.de/cme.

Der Verlag erklärt, dass für die Publikation dieser CME-Fortbildung keine Sponsorengelder an den Verlag fließen.

Für diesen Beitrag wurden von den Autoren keine Studien an Menschen oder Tieren durchgeführt. Für die aufgeführten Studien gelten die jeweils dort angegebenen ethischen Richtlinien.

Open Access. Dieser Artikel wird unter der Creative Commons Namensnennung 4.0 International Lizenz veröffentlicht, welche die Nutzung, Vervielfältigung, Bearbeitung Verbreitung und Wiedergabe in jeglichem Medium und Format erlaubt, sofern Sie den/die ursprünglichen Autor(en) und die Quelle ordnungsgemäßnennen, einen Link zur Creative Commons Lizenz beifügen und angeben, ob Änderungen vorgenommen wurden.

Die in diesem Artikel enthaltenen Bilder und sonstiges Drittmaterial unterliegen ebenfalls der genannten Creative Commons Lizenz, sofern sich aus der Abbildungslegende nichts anderes ergibt. Sofern das betreffende Material nicht unter der genannten Creative Commons Lizenz steht und die betreffende Handlung nicht nach gesetzlichen Vorschriften erlaubt ist, ist für die oben aufgeführten Weiterverwendungen des Materials die Einwilligung des jeweiligen Rechteinhabers einzuholen.

Weitere Details zur Lizenz entnehmen Sie bitte der Lizenzinformation auf http:// creativecommons.org/licenses/by/4.0/deed.de.

\section{Literatur}

1. https://www.awmf.org/leitlinien/detail/ll/015-085.html, zugegriffen 15. Dez. 2019

2. Barratt CLR, Björndahl L, DeJonghe CJ et al (2017) The diagnosis of male infertility: an analysis of the evidence to support the development of global WHO guidancechallenges and future research opportunities. Hum Reprod Update 23:660-680

3. Krausz C, Hoefsloot L, Simoni M, Tüttelmann F (2014) European Academy of Andrology; European Molecular Genetics Quality Network. EAA/EMQN best practice guidelines for molecular diagnosis of Y-chromosomal microdeletions: state-of-the-art 2013. Andrology 2:5-19

4. Stouffs K, Vloeberghs V, Gheldof A et al (2017) Are AZFb deletions always incompatible with sperm production? Andrology 5:691-694

5. Tüttelmann F, Gromoll J, Kliesch S (2008) Genetik der männlichen Infertilität. Urologe 47:1561-1567

6. Dul EC, Van Ravenswaaij-Arts CMA, Groen Het al (2010) Who should be screened for chromosomal abnormalities before ICSI treatment? Hum Reprod 25:2673-2677
7. Gekas J, Thepot F, Turleau C et al (2001) Chromosomal factors of infertility in candidate couples for ICSI: an equal risk of constitutional aberrations in women and men. Hum Reprod 16:82-90

8. Donker RB, Vloeberghs V, Groen H, Tournaye H et al (2017) Chromosomal abnormalities in 1663 infertile men with azoospermia: the clinical consequences. Hum Reprod 32:2574-2580

9. Harper JC, Aittomäkki K, Borry P et al (2018) Recent developments in genetics and medically assisted reproduction: from research to clinical applications. Eur J Hum Genet 26:12-33

10. Tüttelmann F, Ruckert C, Röpke A et al (2018) Disorders of spermatogenesis: perspectives for novel genetic diagnostics after 20 years of unchanged routine. medgen 30:12-20

11. Schwarzer JU, Schwarz M (2012) Significance of CFTR gene mutations in patients with congenital aplasia of vas deferens with special regard to renal aplasia. Andrologia 44:305-307

12. Patat O, Pagin A, Siegfried A et al (2016) Truncating mutations in the adhesion $G$ protein-coupled receptor $\mathrm{G} 2$ gene ADGRG 2 cause an X-linked congenital bilateral absence of vas deferens. Am J Hum Genet 99:437-442

13. Tüttelmann F, Gromoll J (2010) Novel genetic aspects of Klinefelter's syndrome. Mol Hum Reprod 16:386-395

14. Nieschlag E (2013) Klinefelter syndrome: the commonest form of hypogonadism, but often overlooked or untreated. Dtsch Arztebl Int 110:347-353

15. Corona G, Pizzocaro A, Lanfranco F et al (2017) Sperm recovery and ICSI outcomes in Klinefelter syndrome: a systematic review and meta-analysis. Hum Reprod Update 23:265-275

16. Bonomi M, Vezzoli V, Krausz C et al (2018) Characteristics of a nationwide cohort of patients presenting with isolated hypogonadotropic hypogonadism $(\mathrm{IHH})$. Eur J Endocrinol 178:23-32

17. Boehm U, Bouloux P-M, Dattani MT et al (2015) European Consensus Statement on congenital hypogonadotropic hypogonadism - pathogenesis, diagnosis and treatment. Nat Rev Endocrinol 11:547-564

18. Franasiak JM, Forman EJ, Hong KH et al (2014) The nature of aneuploidy with increasing age of the female partner: a review of 15,169 consecutive trophectoderm biopsies evaluated with comprehensive chromosomal screening. Fertil Steril 101:656-663.e1

19. Jahrbuch 2016 Deutsches IVF Register (2017) JReprod Endokrin 6:275-305

20. Qin Y, Jiao X, Simpson JL et al (2015) Genetics of primary ovarian insufficiency: new developments and opportunities. Hum Reprod Update 21:787-808

21. Pasquino AM, Passeri F, Pucarelli I et al (1997) Spontaneous pubertal development in Turner's syndrome. J Clin Endocrinol Metab 82:1810-1813

22. Homer L, Le Martelot MT, Morel F et al (2010) 45,X/46,XX mosaicism below $30 \%$ of aneuploidy: clinical implications in adult women from a reproductive medicine unit. Eur JEndocrinol 162:617-623

23. Grynberg M, Bidet M, Bernard J et al (2016) Fertility preservation in Turner syndrome. Fertil Steril 105:13-19

24. Shankar RK, Backeljauw PF (2018) Current best practice in the management of Turner syndrome. Ther Adv Endocrinol Metab 9:33-40

25. Mercadal AB, Imbert R, Demeestere l et al (2011) Pregnancy outcome after oocyte donation in patients with Turner's syndrome and partial X monosomy. Hum Reprod 26:2061-2068

26. Tartaglia NR, Howell S, Sutherland A et al (2010) A review of trisomy X (47, XXX). Orph JRare Dis 5:8

27. Bagni C, Tassone F, Neri G et al (2012) Fragile $X$ syndrome: causes, diagnosis, mechanisms, and therapeutics. J Clin Invest 122(12):4314-4322

28. Nelson LM (2009) Primary ovarian insufficiency. N Eng J Med 360:606-614

29. Layman LC (2013) The genetic basis of female reproductive disorders: etiology and clinical testing. Mol Cell Endocrinol 370:138-148

30. http://www.awmf.org/leitlinien/detail/Il/174-013.html,zugegriffen 15.Dez. 2019

31. Riccaboni A, Lalatta F, Caliari I et al (2008) Genetic screening in 2,710 infertile candidate couples for assisted reproductive techniques: results of application of Italian guidelines for the appropriate use of genetic tests. Fertil Steril 89:800-808

32. Foresta C, Ferlin A, Gianaroli L et al (2002) Guidelines for the appropriate use of genetic tests in infertile couples. Eur J Hum Genet 10:303-312

33. ACOG Committee on Obstetric Practice, ACOG Committee on Gynecologic Practice, ACOG Committee on Genetics. (2005) ACOG Committee Opinion \#324: Perinatal risks associated with assisted reproductive technology. Obstet Gynecol 106:1143-1146

34. https://www.nice.org.uk/guidance/cg156, zugegriffen 15.Dez. 2019

35. Okun N, Sierra S (2014) Pregnancy outcomes after assisted human reproduction. JObstet Gynaecol Can 36:64-83

36. Practice Committee of the American Society for Reproductive Medicine (2015) Diagnostic evaluation of the infertile male: a committee opinion. Fertil Steril 103:e18-e25 
Welche genetische Untersuchung empfehlen Sie einem Paar mit unerfülltem Kinderwunsch ohne auffällige Vorbefunde?

O Bestimmung des CGG-Repeats im FMR1Gen

O Sequenzierung des CFTR-Gens

O Darstellung der Azoospermiefaktorregionen

O Analyse des CYP21A2-Gens

O Chromosomenanalyse

? Welche Diagnostik veranlassen Sie bei einem Mann mit sonographischem Nachweis einer Fehlanlage beider Samenleiter und Azoospermie in einem weiteren Schritt?

O Magnetresonanztomographie der Hypophyse

O CFTR-Analyse

Öberprüfung des Geruchsinns

○ Messung des Hodenvolumens

O Ausschluss Y-Mikrodeletion

Bei einer Frau mit Ovarialinsuffizienz wird eine Prämutation von 80 CGGRepeats im FMR1-Gen festgestellt. Wie hoch ist das Risiko, dass ein künftiger Sohn an einem Fragiles-X-Syndrom erkrankt?

O $10-20 \%$

○ $20-30 \%$

○ $30-40 \%$

○ $50-60 \%$

○ $60-70 \%$
? Die Chromosomenanalyse eines Paars mit unerfülltem Kinderwunsch zeigt eine balancierte Translokation $(2 ; 6)$ bei der Frau. Was bedeutet diese Diagnose?

O In einer Schwangerschaft ist eine pränatale Chromosomenanalyse indiziert.

O Die Frau hat ein hohes Risiko für angeborene Organfehlbildungen.

O Das Paar wird keine gesunden Kinder bekommen können.

○ Bei der Frau ist eine primäre Ovarialinsuffizienz zu erwarten.

○ Eine andrologische Untersuchung des Mannes ist nicht notwendig.

? Ab welchem mütterlichen Alter ist die Mehrzahl der Blastozysten genetisch so weit geschädigt, dass eine künstliche Befruchtung wenig aussichtsreich ist?

20-25 Jahre

O 25-30 Jahre

○ 30-35 Jahre

O 35-40 Jahre

O 40-45 Jahre

? Bei einer Frau mit Hirsutismus und Zyklusstörungen wird eine homozygote Mutation im CYP21A2-Gen nachgewiesen. Welche Diagnose ist damit gesichert?

O Fragiles-X-Syndrom

Klinefelter-Syndrom

O Kallmann-Syndrom
Adrenogenitales Syndrom

O Turner-Syndrom

? Welche genetische Untersuchung ist bei einem Mann mit hypogonadotropem Hypogonadismus sinnvoll?

O Analyse der CGG-Repeats im FMR1-Gen

O Chromosomenanalyse

O Analyse der $\mathrm{CHH}$-Gene

O Analyse auf Azoospermiefaktordeletion

O Analyse des CFTR-Gens

? Bei welchem genetischen Befund ist nicht mit einer erfolgreichen Anwendung von Techniken der assistierten Reproduktion zu rechnen?

O Azoospermiefaktor-a-Deletion

O Karyotyp 47,XXX

O Kallmann-Syndrom

O Karyotyp 47,XXY

○ Azoospermiefaktor-c-Deletion

Es stellen sich ein Mann mit zystischer Fibrose und seine gesunde Partnerin mit unerfülltem Kinderwunsch vor. Die genetische Untersuchung zeigt bei der Frau eine heterozygote CFTRMutation. Wie hoch ist das Risiko einer zystischen Fibrose bei künftigen Kindern einzuordnen?

O $5 \%$

○ $10 \%$

○ $25 \%$

○ $50 \%$

○ $75 \%$

\section{Informationen zur zertifizierten Fortbildung}

Diese Fortbildung wurde von der Ärztekammer Nordrhein für das „Fortbildungszertifikat der Ärztekammer" gemäß 55 ihrer Fortbildungsordnung mit 3 Punkten (Kategorie D) anerkannt und ist damit auch für andere Ärztekammern anerkennungsfähig.
Anerkennung in Österreich: Für das Diplom-Fortbildungs-Programm (DFP) werden die von deutschen Landesärztekammern anerkannten Fortbildungspunkte aufgrund der Gleichwertigkeit im gleichen Umfang als DFP-Punkte anerkannt (§ 14, Abschnitt 1, Verordnung über ärztliche Fortbildung, Österreichische Ärztekammer (ÖÄK) 2013).
Hinweise zur Teilnahme:

- Die Teilnahme an dem zertifizierten Kurs ist nur online auf www.springermedizin.de/cme möglich.

- Der Teilnahmezeitraum beträgt 12 Monate. Den Teilnahmeschluss finden Sie online beim Kurs.

- Die Fragen und ihre zugehörigen Antwortmöglichkeiten werden online in zufälliger Reihenfolge zusammengestellt.
- Pro Frage ist jeweils nur eine Antwort zutreffend.

- Für eine erfolgreiche Teilnahme müssen 70\% der Fragen richtig beantwortet werden.

- Teilnehmen können Abonnenten dieser Fachzeitschrift und e.Med-Abonnenten. 
? Welche Maßnahme ist bei einer jungen Frau mit Turner-Syndrom am ehesten angezeigt, um später einen eigenen Kinderwunsch zu realisieren?

O Chromosomenanalyse des Partners

O Kryokonservierung von Eizellen

O Östrogenersatztherapie

Kontrolle der Gonadotropine

○ Ausschluss von Uterusfehlbildungen

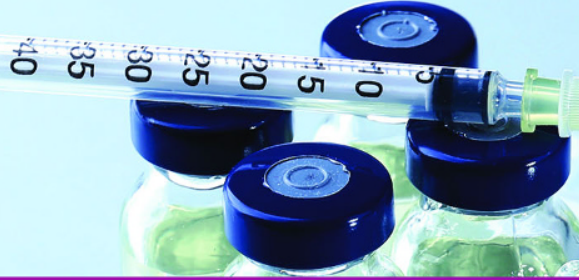

Der neue Kurs: Impfungen

Leitlinienorientiert und fallbasiert

$\checkmark$ Optimiert für Smartphones

$\checkmark$ Zertifiziert mit 3 Punkten

Diesen CME-Kurs finden Sie auf

\section{» DGIM-eAkademie.de}

Das Fortbildungs-Portal der DGIM:

Kostenfrei für alle Mitglieder und e.Med-Abonnenten

․ Alle neuen Kurse sind optimiert für die Teilnahme per Smartphone

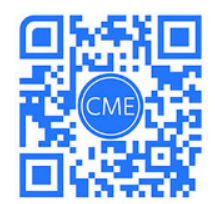

